\section{A COMFORT FOR BED-RIDDEN PATIENTS.} To the Editor of THe Lancet.

Sir,-Through the medium of your columns I am anxious to make known a simple means, productive of great comfort both to the patient and surgeon. A.S-, a patient under my care in the Hackney Union Infirmary, has for some time "passed everything under her," and thereby become a nuisance and cause of complaint to the other patients in the ward. Eleven days ago, I adopted the plan of placing beneath her a calico bag two feet square, partially filled with Irish peat-charcoal, so as to form a sort of cushion and absorbing medium. It has had the happy effect, - which continues even now, without any necessity for changing the charcoal,-of completely neutralizing all unpleasant odour; and if the bed becomes partially wet, all the offensive ingredients are absorbed and neutralized by the charcoal, which thus is a most simple means of remedying a great nuisance, and one that requires the most strict attention, at best, to prevent, and that attention is often difficult, and always expensive, to procure. In cases of incontinence of urine particularly, and, indeed, all attended with fœetid discharges, - cancer, compound fractures, \&c., this plan, or some modification of it, might be adopted with advantage.

I have been informed that some of the same material has been placed in the urinals of the South Western Railway, with equally good result in the prevention of unpleasant odour; and that even after it has been unchanged for some weeks, the fluid that percolates has been found by chemical analysis to contain little or no trace of the organic or saline products of urine. This fact induced me to try it as above.

An argument in favour of its adoption in hospitals and lunatic asylums is, that the peat, after its deodorizing properties are exhausted, becomes more valuable for the purpose of manure, so that its use is without expense.

I am, Sir, your obedient servant,

D. De BerdT Hoveli, F.R.C.S.E.

Five-houses, Clapton, Jan. 31st, 1852.

\section{MEETINGS OF THE MEDICAL SOCIETIES IN} LONDON DURING THE ENSUING WEEK.

Note. - When the day of the month is not specified, no meetings take place.

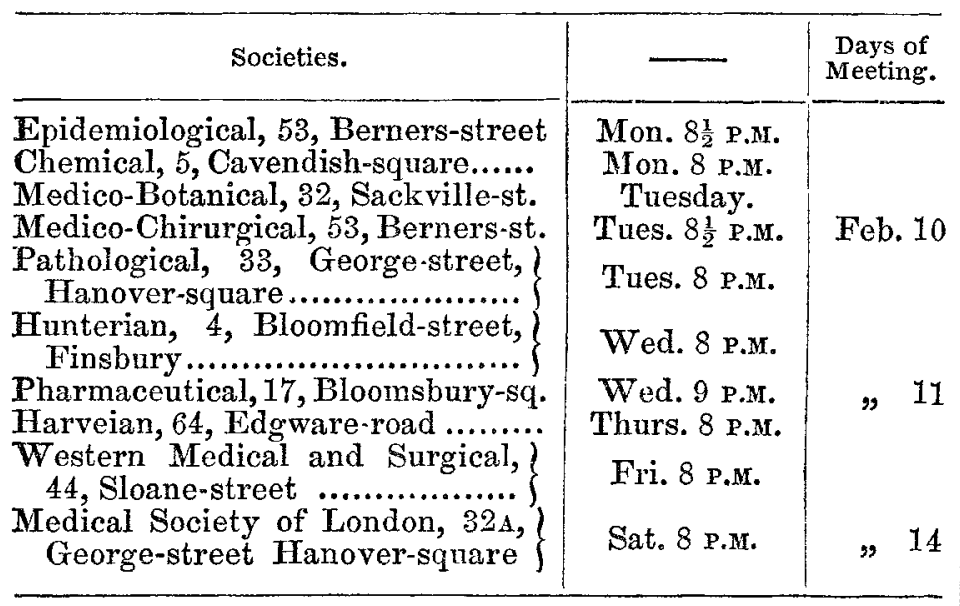

\section{fitedical fines.}

Apothecaries' Hall.-Names of gentlemen who passed their examination in the science and practice of medicine, and received certificates to practise, on

$$
\text { Thursday, Jan. 29th, 1852. }
$$

Cleeve, Frederick Ward, Bradninch, Devon.

HaLls, Thomas EDWard, Broad-street, Horsleydown.

Simpson, Henry, Chester.

King's College Hospital. - According to the plans and specifications of the proposed additions to King's College Hospital, the new buildings will cover the whole of the Green-yard Burial Ground, and also the site of the houses lately pulled down in Carey-street. The excavations for the foundations will be made very deep, in order that the bodies in the graveyard may be removed to a suburban cemetery. It is unnecessary to add that so dense and so populous a neighbourhood demands an hospital of very large extent.
The Dreadnought Seaman's Hospital. - On Wednesday, the annual meeting of Governors was held at their office, King William-street, City, John Wyld, Esq., M.P., and V.P., in the chair. Mr. Cooke read the report, which clearly demonstrated the value and importance of the hospital. During the year, 2242 patients were admitted, and 1642 outpatients were supplied with medical attention, medicines, and medical stores. Of those admitted, sixty were clothed, after being cured, and eleven partially so. During the same period, 1514 were cured, and 105 died. Since the opening of the hospital, in 1821, upwards of 65,587 patients were relieved. Her gracious Majesty subscribed 100 guineas, which generous example was imitated by her royal consort subscribing a similar sum, and becoming one of the vice-patrons. The receipts amounted to $\$ 92982 s .4 d$, and the expenditure to $£ 6137$. The stock figured $£ 3008$. Thanks were voted to the medical and other officers of the past year, who were reelected, and the meeting then separated.

Health of Towns Act. - The inhabitants of Lynn have memorialized to be placed under the superintendence of the Board of Health.

Newcastle-upon-Tyne College of Medrcine.At a convocation held at Durham, on Tuesday, January 27 , the articles of foundation of the Newcastle-upon-Tyne College of Medicine were read and approved by the House. A grace was passed for signifying the approbation of the University by setting the University seal to the above articles of foundation. A grace was passed, in agreement with a provision of the said articles, for giving to the above-mentioned College of Medicine the name of "The Newcastle-on-Tyne College of Medicine in conmexion with the University of Durham."

Fever in Ireland.-We learn, with much regref, that fever is raging throughout Ireland. In the Clonmel Union alone there are 109 fever patients. It is to be hoped that government will take a hint from the past, and adopt immediate and the most prompt measures to prevent a recurrence of the appalling sacrifice of human life that took place two years ago, in that country, from the combined effects of hunger, disease, and neglect. During that awful visitation a large number of medical men, clergymen, and guardians of the poor, fell victims to fever, caught in the discharge of their duties.

Important to Governors of Hospitals.-Mr. Napier, Q. C., applied to the Court of Queen's Bench for a mandamus to compel the Governors of the Limerick Fever Hospital to declare Dr. Griffin elected to the office of physician, vice Dr. Geary, resigned. The learned counsel stated that at an election lately held for a physician to the hospital, Dr.Griffin had the majority of personal votes, but was rejected, as the other candidate had a larger number by proxies. Judge Crampton decided, that as proxies were declared illegal, Dr. Griffin was duly elected, and he, therefore, without hesitation, issued the mandamus.

Corm Prison Infirmary.-From the report of the surgeon of the County Cork prison, for 1851, we find the following results of hospital treatment and expenditure :Number received in hospital, 820 ; daily average number in hospital, 42 ; total number prescribed for, 22,318. Deaths, 68 , viz: fever, 3 ; dysentery, 22 : diarrhoea, 8 ; consumption, 10 ; old age and debility, 25. Cost of each patient, per day, for sustenance and medicine, $3 \frac{1}{2} \mathrm{~d}$.; annual cost of medicine, $£ 65$ 12s. 5 d.

Suffolk Lunatic Asylum. - According to the 14th report, just published by Dr. Kirkman, it appears there were admitted, last year, ninety-one patients. Of that number, fifty-one were cured, thirty-five died. The report stated, that the most beneficial results followed from giving the patients such employment as amused them, and kept their minds engaged.

The Professor of Anatomy, Oxford, resumed his course of lectures on Human Anatomy and Physiology, on Tuesday, Feb. 3, at the Anatomical Schools, at one o'clock.

Liability of Employers for the Medical Expenses of Servants.-At the Wolverhampton County Court, Mr. Kempson, a surgeon, sued Frederick Woodhall, a miner in the employ of Lord Ward, for £33 18s, being his charge for amputating defendant's leg, for attendance, and medicine. Defendant pleaded non debet, inasmuch as he was in Lord Ward's employ at the time of the accident, and that 